STATE OF ALASKA

DEPARTMENT OF NATURAL RESOURCES

Alaska Geologic Materials Center Data Report No. 420

\title{
No. 420
}

Shimer, G., 2013, X-Ray Fluorescence Spectroscopy and ${ }^{40} \mathrm{Ar} /{ }^{39} \mathrm{Ar}$ analyses of core from the Gubik Test \#1, Gubik

Test \#2, Square Lake Test Well \#1, Umiat \#2, Umiat \#9, and Umiat Test Well \#11 wells.

Received September 2013

All data reports may be downloaded free of charge from the DGGS website. 
Bentonite Major Element Geochemistry: X-Ray Fluorescence Spectroscopy

\begin{tabular}{|c|c|c|c|c|c|c|c|c|c|c|c|c|c|}
\hline Sample & $\begin{array}{c}\mathrm{Al}_{2} \mathrm{O}_{3} \\
(\%)\end{array}$ & $\begin{array}{c}\mathrm{Ba} \\
(\mathrm{ppm})\end{array}$ & $\begin{array}{l}\mathrm{CaO} \\
(\%) \\
\end{array}$ & $\begin{array}{c}\mathrm{Cl} \\
(\%) \\
\end{array}$ & $\begin{array}{c}\mathrm{Fe}_{2} \mathrm{O}_{3} \\
(\%)\end{array}$ & $\begin{array}{l}\mathrm{K}_{2} \mathrm{O} \\
(\%) \\
\end{array}$ & $\begin{array}{c}\mathrm{MgO} \\
(\%)\end{array}$ & $\begin{array}{c}\mathrm{MnO} \\
(\%)\end{array}$ & $\begin{array}{c}\mathrm{Na}_{2} \mathrm{O} \\
(\%)\end{array}$ & $\begin{array}{c}\mathrm{P}_{2} \mathrm{O}_{5} \\
(\%) \\
\end{array}$ & $\begin{array}{c}\mathrm{S} \\
(\%) \\
\end{array}$ & $\begin{array}{l}\mathrm{SiO}_{2} \\
(\%)\end{array}$ & $\begin{array}{c}\mathrm{TiO}_{2} \\
(\%) \\
\end{array}$ \\
\hline Umiat 2311 Residue & 22.62 & 1158.35 & 1.88 & 0.04 & 4.07 & 1.03 & 2.85 & 0.02 & 2.48 & 0.17 & -0.01 & 58.35 & 0.92 \\
\hline Umiat 112258 & 21.73 & 1374.00 & 0.43 & 0.02 & 3.63 & 1.36 & 2.37 & 0.01 & 2.94 & 0.07 & 0.01 & 64.49 & 0.57 \\
\hline Umiat 2311 Whole & 23.37 & 1161.27 & 3.58 & 0.03 & 4.23 & 1.09 & 2.81 & 0.03 & 2.23 & 0.18 & 0.01 & 57.67 & 0.94 \\
\hline Umiat 111682 & 20.24 & 1021.59 & 4.18 & 0.02 & 4.70 & 0.92 & 2.41 & 0.09 & 3.27 & 0.08 & 0.26 & 63.03 & 0.66 \\
\hline Umiat 11391 & 18.71 & 413.63 & 0.51 & 0.01 & 4.02 & 0.18 & 2.54 & 0.00 & 3.07 & 0.01 & -0.01 & 72.02 & 0.22 \\
\hline Umiat 111962 & 21.40 & 838.56 & 1.36 & 0.03 & 5.48 & 1.03 & 2.93 & 0.02 & 3.15 & 0.23 & 0.13 & 61.27 & 0.63 \\
\hline Umiat 11345 & 19.67 & 773.63 & 0.72 & 0.01 & 4.96 & 0.21 & 2.49 & 0.00 & 3.30 & 0.01 & 0.04 & 68.80 & 0.35 \\
\hline
\end{tabular}

Bentonite Trace Element Geochemistry: X-Ray Fluorescence Spectroscopy

\begin{tabular}{|c|c|c|c|c|c|c|c|c|c|c|c|c|c|c|c|c|c|c|c|}
\hline Sample & $\begin{array}{c}\mathrm{Ag} \\
(\mathrm{ppm})\end{array}$ & $\begin{array}{c}\text { As } \\
(\mathrm{ppm})\end{array}$ & $\begin{array}{c}\mathrm{Bi} \\
(\mathrm{ppm})\end{array}$ & $\begin{array}{c}\mathrm{Br} \\
(\mathrm{ppm})\end{array}$ & $\begin{array}{c}\mathrm{Cd} \\
(\mathrm{ppm})\end{array}$ & $\begin{array}{c}\mathrm{Ce} \\
(\mathrm{ppm})\end{array}$ & $\begin{array}{c}\text { Co } \\
(\mathrm{ppm})\end{array}$ & $\begin{array}{c}\mathrm{Cr} \\
(\mathrm{ppm})\end{array}$ & $\begin{array}{c}\mathrm{Cs} \\
(\mathrm{ppm})\end{array}$ & $\begin{array}{c}\mathrm{Cu} \\
(\mathrm{ppm})\end{array}$ & $\begin{array}{c}\mathrm{Ga} \\
(\mathrm{ppm})\end{array}$ & $\begin{array}{c}\mathrm{Ge} \\
(\mathrm{ppm})\end{array}$ & $\begin{array}{c}\mathrm{Hf} \\
(\mathrm{ppm})\end{array}$ & $\begin{array}{c}\mathrm{La} \\
(\mathrm{ppm})\end{array}$ & $\begin{array}{c}\mathrm{Mn} \\
(\mathrm{ppm})\end{array}$ & $\begin{array}{c}\text { Mo } \\
(\mathrm{ppm})\end{array}$ & $\begin{array}{l}\mathrm{Nb} \\
(\mathrm{ppm})\end{array}$ & $\begin{array}{l}\mathrm{Nd} \\
(\mathrm{ppm})\end{array}$ & $\begin{array}{l}\mathrm{Ni} \\
(\mathrm{ppm})\end{array}$ \\
\hline Umiat 2311 Whole & -3.84 & -10.49 & -2.45 & -1.90 & 0.62 & 91.59 & 9.73 & 106.94 & 2.77 & 15.28 & 28.50 & 0.11 & 5.85 & 43.54 & 218.77 & 1.58 & 5.88 & 32.04 & 30.19 \\
\hline Umiat 11391 & -1.64 & -9.16 & -1.64 & -1.71 & 0.80 & 115.55 & 2.05 & 3.51 & 2.08 & 4.39 & 26.07 & -0.35 & 7.00 & 57.82 & 18.07 & 0.48 & 7.85 & 51.20 & 2.41 \\
\hline Umiat 11345 & -2.89 & -6.52 & -0.94 & -2.05 & 0.96 & 109.16 & 3.65 & 2.83 & 1.29 & 4.78 & 27.93 & -0.19 & 7.28 & 48.78 & 21.06 & 1.23 & 12.47 & 49.98 & 12.25 \\
\hline Umiat 2311 Residue & -4.84 & -8.13 & -2.05 & -1.45 & 1.89 & 114.96 & 10.16 & 108.79 & 1.29 & 14.81 & 29.57 & -0.19 & 6.45 & 57.56 & 147.21 & 1.06 & 5.41 & 45.32 & 32.13 \\
\hline 111962 & -1.50 & -5.95 & -2.18 & -1.96 & 2.80 & 74.57 & 5.97 & 21.21 & 5.43 & 8.08 & 24.85 & -0.17 & 4.44 & 43.85 & 159.27 & 2.19 & 6.84 & 29.93 & 7.54 \\
\hline Umiat 111682 & -3.11 & -0.17 & -2.00 & -1.99 & 2.03 & 119.18 & 4.10 & 8.88 & 11.48 & 10.20 & 22.85 & -0.20 & 5.87 & 68.52 & 660.78 & 4.13 & 8.93 & 58.09 & 4.64 \\
\hline Umiat 112258 & -2.77 & -6.81 & -0.51 & -1.57 & -0.44 & 92.15 & 3.34 & 26.95 & 4.67 & 14.55 & 28.08 & -0.09 & 10.23 & 45.56 & 60.14 & 2.40 & 11.77 & 36.61 & 10.23 \\
\hline
\end{tabular}

\begin{tabular}{|c|c|c|c|c|c|c|c|c|c|c|c|c|c|c|c|c|c|c|c|c|}
\hline Sample & $\begin{array}{c}\mathrm{Pb} \\
(\mathrm{ppm}) \\
\end{array}$ & $\begin{array}{c}\mathrm{Rb} \\
(\mathrm{ppm}) \\
\end{array}$ & $\begin{array}{c}\mathrm{Sb} \\
(\mathrm{ppm}) \\
\end{array}$ & $\begin{array}{c}\mathrm{Sc} \\
(\mathrm{ppm}) \\
\end{array}$ & $\begin{array}{c}\mathrm{Se} \\
(\mathrm{ppm})\end{array}$ & $\begin{array}{c}\mathrm{Sm} \\
(\mathrm{ppm}) \\
\end{array}$ & $\begin{array}{c}\text { Sn } \\
(\mathrm{ppm}) \\
\end{array}$ & $\begin{array}{c}\mathrm{Sr} \\
(\mathrm{ppm}) \\
\end{array}$ & $\begin{array}{c}\mathrm{Ta} \\
(\mathrm{ppm}) \\
\end{array}$ & $\begin{array}{c}\mathrm{Te} \\
(\mathrm{ppm})\end{array}$ & $\begin{array}{c}\text { Th } \\
(\mathrm{ppm})\end{array}$ & $\begin{array}{c}\mathrm{Tl} \\
(\mathrm{ppm}) \\
\end{array}$ & $\begin{array}{c}\mathrm{U} \\
(\mathrm{ppm}) \\
\end{array}$ & $\begin{array}{c}\mathrm{V} \\
(\mathrm{ppm}) \\
\end{array}$ & $\begin{array}{c}\text { W } \\
(\mathrm{ppm}) \\
\end{array}$ & $\begin{array}{c}\mathrm{Y} \\
(\mathrm{ppm}) \\
\end{array}$ & $\begin{array}{c}\mathrm{Yb} \\
(\mathrm{ppm})\end{array}$ & $\begin{array}{c}\mathrm{Zn} \\
(\mathrm{ppm}) \\
\end{array}$ & $\begin{array}{c}\mathrm{Zn} \\
(\mathrm{ppm}) \\
\end{array}$ & $\begin{array}{l}\mathrm{Zr} \\
(\mathrm{ppm})\end{array}$ \\
\hline Umiat 2311 Whole & 41.24 & 39.33 & -3.92 & 10.65 & -2.09 & 3.36 & 9.19 & 221.15 & 2.50 & -6.41 & 35.39 & 7733.39 & -4.10 & 10.51 & 73.13 & 0.62 & 16.30 & 3.86 & 70.30 & 216.7 \\
\hline Umiat 11391 & 36.57 & 9.54 & -1.81 & 10.75 & -1.39 & 5.96 & 10.10 & 218.60 & 1.59 & -2.85 & 25.51 & 1567.15 & -4.21 & 9.91 & 4.31 & 0.70 & 44.18 & 5.31 & 42.74 & 200.7 \\
\hline Umiat 11345 & 30.89 & 9.56 & 0.54 & 14.49 & -1.53 & 9.91 & 7.19 & 331.84 & -0.14 & -3.73 & 25.50 & 2343.70 & -2.82 & 12.02 & 10.67 & 1.88 & 38.56 & 2.83 & 77.54 & 282.5 \\
\hline Umiat 2311 Residue & 46.37 & 39.91 & -1.02 & 12.40 & -1.80 & 1.44 & 9.53 & 222.09 & 1.44 & -0.50 & 35.99 & 7855.94 & -3.97 & 13.90 & 76.80 & -0.13 & 18.51 & 2.80 & 80.96 & 237.1 \\
\hline Umiat 111962 & 28.77 & 37.31 & -1.78 & 8.10 & -2.49 & 2.39 & 8.25 & 356.59 & 1.79 & -1.86 & 20.13 & 5097.02 & -4.29 & 8.53 & 49.63 & 0.37 & 15.87 & 3.30 & 88.00 & 188.7 \\
\hline Umiat 111682 & 24.63 & 46.48 & -2.99 & 14.86 & -1.36 & 7.74 & 7.04 & 361.31 & 1.62 & -3.85 & 29.91 & 5291.84 & -4.00 & 11.78 & 28.80 & 3.91 & 37.33 & 3.68 & 105.41 & 309.6 \\
\hline Umiat 112258 & 51.78 & 47.52 & 0.14 & 12.50 & -1.23 & 3.73 & 9.98 & 255.87 & 1.73 & -2.14 & 31.61 & 3725.19 & -3.21 & 12.39 & 26.22 & -0.81 & 36.08 & 4.65 & 91.96 & 326 \\
\hline
\end{tabular}


Sandsone Trace Element Geochemistry: X-Ray Fluorescence Spectroscopy

\begin{tabular}{|c|c|c|c|c|c|c|c|c|c|c|c|c|c|c|c|c|c|c|c|c|}
\hline Sample & $\begin{array}{c}\mathrm{Ag} \\
\text { (ppm) }\end{array}$ & $\begin{array}{c}\text { As } \\
(\mathrm{ppm})\end{array}$ & $\begin{array}{c}\mathrm{Ba} \\
(\mathrm{ppm})\end{array}$ & $\begin{array}{c}\mathrm{Bi} \\
(\mathrm{ppm}) \\
\end{array}$ & $\begin{array}{c}\mathrm{Br} \\
(\mathrm{ppm})\end{array}$ & $\begin{array}{c}\mathrm{Cd} \\
(\mathrm{ppm}) \\
\end{array}$ & $\begin{array}{c}\mathrm{Ce} \\
\text { (ppm) }\end{array}$ & $\begin{array}{c}\text { Co } \\
(\mathrm{ppm})\end{array}$ & $\begin{array}{c}\mathrm{Cr} \\
(\mathrm{ppm})\end{array}$ & $\begin{array}{c}\mathrm{Cs} \\
(\mathrm{ppm})\end{array}$ & $\begin{array}{c}\mathrm{Cu} \\
(\mathrm{ppm})\end{array}$ & $\begin{array}{c}\mathrm{Ga} \\
(\mathrm{ppm}) \\
\end{array}$ & $\begin{array}{c}\mathrm{Ge} \\
(\mathrm{ppm})\end{array}$ & $\begin{array}{c}\mathrm{Hf} \\
(\mathrm{ppm})\end{array}$ & $\begin{array}{c}\mathrm{La} \\
(\mathrm{ppm})\end{array}$ & $\begin{array}{c}\mathrm{Mn} \\
(\mathrm{ppm})\end{array}$ & $\begin{array}{l}\text { Mo } \\
(\mathrm{ppm})\end{array}$ & $\begin{array}{l}\mathrm{Nb} \\
(\mathrm{ppm})\end{array}$ & $\begin{array}{l}\mathrm{Nd} \\
(\mathrm{ppm})\end{array}$ & $\begin{array}{l}\mathrm{Ni} \\
(\mathrm{ppm}) \\
\end{array}$ \\
\hline Umiat 9410 & -6.15 & -1.52 & 525.36 & -2.36 & -1.58 & -3.69 & 58.75 & 18.13 & 179.90 & 3.99 & 17.71 & 14.47 & 1.00 & 4.84 & 33.09 & 224.38 & 5.93 & 15.89 & 27.26 & 51.4 \\
\hline Umiat 9591 & -5.57 & -1.63 & 476.89 & -2.04 & -2.18 & -5.43 & 59.72 & 14.38 & 111.39 & 9.49 & 34.99 & 14.31 & 0.62 & 3.40 & 22.62 & 639.19 & 3.85 & 12.29 & 24.35 & 46.3 \\
\hline Umiat 91080 & -4.37 & -6.47 & 416.32 & -1.98 & -1.27 & -1.67 & 26.93 & 17.35 & 136.18 & 2.45 & 13.74 & 10.37 & 1.13 & 4.04 & 9.72 & 263.45 & 5.95 & 9.36 & 11.17 & 43.82 \\
\hline Umiat 9699 & -4.13 & 17.49 & 438.67 & -2.90 & -1.84 & -1.57 & 46.58 & 15.57 & 167.62 & 4.00 & 19.38 & 11.47 & 1.47 & 4.96 & 11.80 & 218.67 & 6.05 & 11.72 & 22.69 & 36.69 \\
\hline Umiat 9919 & -5.44 & -5.31 & 658.25 & -2.67 & -1.77 & -2.49 & 21.97 & 13.13 & 105.02 & 1.12 & 10.54 & 7.36 & 0.38 & 3.02 & 6.08 & 540.85 & 4.62 & 6.55 & 7.06 & 32.64 \\
\hline Umiat 91150 & -3.89 & 5.05 & 575.87 & -3.70 & -1.68 & -2.04 & 50.28 & 17.08 & 131.37 & 7.84 & 21.10 & 14.95 & 0.85 & 5.53 & 18.01 & 236.84 & 5.09 & 12.97 & 20.90 & 45.76 \\
\hline
\end{tabular}

\begin{tabular}{|c|c|c|c|c|c|c|c|c|c|c|c|c|c|c|c|c|c|c|c|c|}
\hline $\begin{array}{l}\text { Continued } \\
\text { Sample } \\
\end{array}$ & $\begin{array}{c}\mathrm{Pb} \\
(\mathrm{ppm})\end{array}$ & $\begin{array}{c}\mathrm{Rb} \\
(\mathrm{ppm})\end{array}$ & $\begin{array}{c}\mathrm{Sb} \\
(\mathrm{ppm})\end{array}$ & $\begin{array}{c}\mathrm{Sc} \\
(\mathrm{ppm})\end{array}$ & $\begin{array}{c}\mathrm{Se} \\
(\mathrm{ppm})\end{array}$ & $\begin{array}{c}\mathrm{Sm} \\
(\mathrm{ppm})\end{array}$ & $\begin{array}{c}\mathrm{Sn} \\
(\mathrm{ppm})\end{array}$ & $\begin{array}{c}\mathrm{Sr} \\
(\mathrm{ppm})\end{array}$ & $\begin{array}{c}\mathrm{Ta} \\
(\mathrm{ppm}) \\
\end{array}$ & $\begin{array}{c}\mathrm{Te} \\
(\mathrm{ppm}) \\
\end{array}$ & $\begin{array}{c}\text { Th } \\
(\mathrm{ppm})\end{array}$ & $\begin{array}{c}\mathrm{TiO} 2 \\
(\mathrm{ppm}) \\
\end{array}$ & $\begin{array}{c}\mathrm{Tl} \\
(\mathrm{ppm}) \\
\end{array}$ & $\begin{array}{c}\mathrm{U} \\
(\mathrm{ppm}) \\
\end{array}$ & $\begin{array}{c}\mathrm{V} \\
(\mathrm{ppm}) \\
\end{array}$ & $\begin{array}{c}\mathrm{W} \\
(\mathrm{ppm})\end{array}$ & $\begin{array}{c}\mathrm{Y} \\
(\mathrm{ppm}) \\
\end{array}$ & $\begin{array}{c}\mathrm{Yb} \\
(\mathrm{ppm})\end{array}$ & $\begin{array}{c}\mathrm{Zn} \\
(\mathrm{ppm})\end{array}$ & $\begin{array}{l}\mathrm{Zr} \\
(\mathrm{ppm}) \\
\end{array}$ \\
\hline Umiat 9410 & 12.43 & 71.76 & -1.77 & 14.35 & -1.95 & -2.42 & 3.55 & 78.73 & 0.68 & -5.41 & 12.71 & 9628.39 & -4.63 & 7.58 & 134.19 & 1.32 & 28.68 & 4.30 & 101.32 & 271.2 \\
\hline Umiat 9591 & 14.20 & 75.95 & 0.49 & 18.44 & -1.17 & 4.88 & -0.05 & 136.58 & 2.15 & 0.14 & 10.21 & 7997.23 & -3.15 & 5.16 & 129.82 & 3.35 & 26.31 & 3.98 & 98.575 & 196.7 \\
\hline Umiat 91080 & 8.97 & 57.46 & 1.24 & 11.43 & -1.85 & -0.33 & 3.78 & 87.53 & 1.35 & -1.24 & 6.36 & 6309.23 & -3.92 & 4.37 & 103.14 & 1.35 & 18.31 & 4.69 & 75.054 & 198.9 \\
\hline Umiat 9699 & 10.33 & 60.05 & -0.50 & 9.44 & -2.02 & 3.94 & 0.01 & 94.85 & -0.99 & 2.43 & 7.66 & 8457.77 & -4.79 & 4.31 & 135.12 & -2.00 & 20.08 & 3.25 & 75.135 & 308.6 \\
\hline Umiat 9919 & 4.17 & 44.51 & 3.93 & 21.84 & -1.75 & 2.94 & 1.43 & 279.92 & -1.49 & 0.84 & 4.30 & 4591.73 & -3.38 & 3.29 & 73.70 & -0.69 & 14.54 & 3.44 & 49.979 & 135 \\
\hline Umiat 91150 & 14.05 & 90.64 & -3.10 & 12.77 & -1.60 & 12.30 & 1.38 & 122.56 & 1.99 & 0.20 & 11.59 & 8099.60 & -4.04 & 4.84 & 142.20 & 2.57 & 27.81 & 3.53 & 91.717 & 307.8 \\
\hline
\end{tabular}




\section{UM11-345 SAN\#L1}

Weighted average of $\mathrm{J}$ from standards $=4.215 \mathrm{e}-03+/-2.776 \mathrm{e}-05$

\begin{tabular}{|c|c|c|c|c|c|c|c|c|c|c|c|c|c|c|c|c|}
\hline $\begin{array}{l}\text { Laser Power } \\
(\mathrm{mW})\end{array}$ & $\begin{array}{l}\text { Cumulative } \\
\text { 39Ar }\end{array}$ & $\begin{array}{l}40 \mathrm{Ar} / 39 \mathrm{Ar} \\
\text { measured }\end{array}$ & $+/-$ & $\begin{array}{l}\text { 37 Ar/39Ar } \\
\text { measured }\end{array}$ & $+/-$ & $\begin{array}{l}36 \mathrm{Ar} / 39 \mathrm{Ar} \\
\text { measured }\end{array}$ & $+/-$ & $\begin{array}{l}\text { \% Atmospheric } \\
40 \mathrm{Ar}\end{array}$ & $\mathrm{Ca} / \mathrm{K}$ & $+/-$ & $\mathrm{C} / / \mathrm{K}$ & $+/-$ & $40^{*} / 39 \mathrm{~K}$ & $+/-$ & $\begin{array}{l}\text { Age } \\
\text { (Ma) }\end{array}$ & $\begin{array}{l}+/- \\
\text { (Ma) } \\
\end{array}$ \\
\hline 500 & 0.0115 & 73.01065 & 1.23884 & 0.44072 & 0.00822 & 0.21105 & 0.0036 & 85.40549 & 0.80891 & 0.0151 & 0.00295 & 0.00027 & 10.65453 & 1.1614 & 79.25 & 8.45 \\
\hline 750 & 0.0284 & 30.54076 & 0.43178 & 0.64837 & 0.01006 & 0.06202 & 0.00131 & 59.88706 & 1.19022 & 0.01848 & 0.00285 & 0.00016 & 12.2445 & 0.44903 & 90.79 & 3.25 \\
\hline 1000 & 0.0474 & 23.03402 & 0.34502 & 1.00661 & 0.01764 & 0.03403 & 0.00099 & 43.3571 & 1.84831 & 0.03242 & 0.00191 & 0.00023 & 13.0396 & 0.36906 & 96.53 & 2.66 \\
\hline 1500 & 0.0958 & 18.42292 & 0.33338 & 0.9284 & 0.01678 & 0.02025 & 0.00053 & 32.11408 & 1.70461 & 0.03083 & 0.00132 & 0.00009 & 12.49461 & 0.30601 & 92.59 & 2.21 \\
\hline 2000 & 0.1496 & 16.48898 & 0.27132 & 0.96406 & 0.01674 & 0.01337 & 0.00042 & 23.51693 & 1.77013 & 0.03075 & 0.00108 & 0.00009 & 12.59715 & 0.2658 & 93.33 & 1.92 \\
\hline 2500 & 0.21 & 16.53061 & 0.28982 & 0.91414 & 0.01453 & 0.01276 & 0.00036 & 22.40179 & 1.67841 & 0.02669 & 0.00112 & 0.00007 & 12.8127 & 0.27579 & 94.89 & 1.99 \\
\hline 3000 & 0.2684 & 14.5955 & 0.25229 & 0.99243 & 0.01699 & 0.00666 & 0.00028 & 12.94374 & 1.82226 & 0.03123 & 0.00109 & 0.00008 & 12.68935 & 0.24783 & 94 & 1.79 \\
\hline 5000 & 0.4968 & 14.07138 & 0.19952 & 0.72006 & 0.01249 & 0.00469 & 0.00011 & 9.43986 & 1.32188 & 0.02294 & 0.0007 & 0.00007 & 12.72265 & 0.18584 & 94.24 & 1.34 \\
\hline 9000 & 0.8034 & 14.46213 & 0.20881 & 0.6416 & 0.01108 & 0.00586 & 0.00012 & 11.62485 & 1.17778 & 0.02036 & 0.00039 & 0.00005 & 12.76048 & 0.19027 & 94.51 & 1.37 \\
\hline 9001 & 1 & 14.26377 & 0.20535 & 0.62157 & 0.01044 & 0.00499 & 0.00012 & 10.00582 & 1.141 & 0.01917 & 0.00038 & 0.00005 & 12.81547 & 0.19029 & 94.91 & 1.37 \\
\hline Integrated & & 15.87701 & 0.10228 & 0.72849 & 0.00544 & 0.01083 & 0.00009 & 19.82411 & 1.33736 & 0.00999 & 0.00073 & 0.00003 & 12.71227 & 0.08811 & 94.17 & 0.88 \\
\hline
\end{tabular}




\section{UM11-391 BI\#L1}

Weighted average of $\mathrm{J}$ from standards $=4.215 \mathrm{e}-03+/-2.776 \mathrm{e}-05$

\begin{tabular}{|c|c|c|c|c|c|c|c|c|c|c|c|c|c|c|c|c|}
\hline $\begin{array}{l}\text { Laser Power } \\
(\mathrm{mW})\end{array}$ & $\begin{array}{l}\text { Cumulative } \\
\text { 39Ar }\end{array}$ & $\begin{array}{l}\begin{array}{l}40 \mathrm{Ar} / 39 \mathrm{Ar} \\
\text { measured }\end{array} \\
\end{array}$ & $+/-$ & $\begin{array}{l}\text { 37 Ar } / 39 \mathrm{Ar} \\
\text { measured }\end{array}$ & $+/-$ & $\begin{array}{l}\text { 36Ar/39Ar } \\
\text { measured }\end{array}$ & $+/-$ & $\begin{array}{l}\text { \% Atmospheric } \\
40 \mathrm{Ar}\end{array}$ & $\mathrm{Ca} / \mathrm{K}$ & $+/-$ & $\mathrm{Cl} / \mathrm{K}$ & $+/-$ & $40^{* / 39 K}$ & $+/-$ & $\begin{array}{l}\text { Age } \\
\text { (Ma) }\end{array}$ & $\begin{array}{l}+/- \\
\text { (Ma) }\end{array}$ \\
\hline 300 & 0.0203 & 748.85129 & 9.40603 & 0.57051 & 0.00872 & 2.45798 & 0.04344 & 96.99036 & 1.04723 & 0.01602 & 0.03149 & 0.00228 & 22.54595 & 10.47456 & 163.78 & 72.74 \\
\hline 500 & 0.0443 & 323.00231 & 3.58431 & 5.65562 & 0.08836 & 1.01577 & 0.01453 & 92.79229 & 10.4189 & 0.16343 & 0.03995 & 0.00119 & 23.37228 & 3.5394 & 169.51 & 24.5 \\
\hline 700 & 0.0758 & 159.0748 & 1.54102 & 0.40884 & 0.00698 & 0.47681 & 0.00573 & 88.56892 & 0.75039 & 0.01281 & 0.03656 & 0.001 & 18.18583 & 1.48307 & 133.24 & 10.47 \\
\hline 900 & 0.1166 & 110.15761 & 1.42821 & 0.31279 & 0.00487 & 0.30795 & 0.00489 & 82.60668 & 0.57406 & 0.00893 & 0.03247 & 0.00077 & 19.15913 & 1.13248 & 140.1 & 7.97 \\
\hline 1100 & 0.1655 & 70.34806 & 0.91483 & 0.0664 & 0.00211 & 0.17682 & 0.00285 & 74.29794 & 0.12183 & 0.00388 & 0.02525 & 0.00058 & 18.07411 & 0.70376 & 132.45 & 4.97 \\
\hline 1300 & 0.2134 & 46.67508 & 0.80847 & 0.04741 & 0.00145 & 0.10014 & 0.0016 & 63.42844 & 0.087 & 0.00267 & 0.02346 & 0.00054 & 17.05951 & 0.65667 & 125.27 & 4.66 \\
\hline 1500 & 0.2592 & 41.99756 & 0.65124 & 0.09417 & 0.00217 & 0.08026 & 0.00117 & 56.49217 & 0.17279 & 0.00399 & 0.02897 & 0.00059 & 18.26052 & 0.5616 & 133.77 & 3.97 \\
\hline 1750 & 0.3112 & 37.91312 & 0.63689 & 0.09244 & 0.00196 & 0.0687 & 0.00126 & 53.56494 & 0.16962 & 0.00359 & 0.02871 & 0.00058 & 17.59234 & 0.56286 & 129.05 & 3.98 \\
\hline 2000 & 0.3686 & 35.47469 & 0.61543 & 0.0692 & 0.00143 & 0.06128 & 0.001 & 51.07233 & 0.12697 & 0.00262 & 0.02616 & 0.00058 & 17.34326 & 0.51138 & 127.28 & 3.62 \\
\hline 2250 & 0.4133 & 33.10117 & 0.53995 & 0.05575 & 0.00185 & 0.05292 & 0.00102 & 47.27148 & 0.1023 & 0.0034 & 0.02336 & 0.00044 & 17.43878 & 0.48411 & 127.96 & 3.43 \\
\hline 2500 & 0.4617 & 31.51864 & 0.48408 & 0.08511 & 0.00208 & 0.04768 & 0.001 & 44.72 & 0.15617 & 0.00382 & 0.02238 & 0.00043 & 17.40813 & 0.47241 & 127.74 & 3.35 \\
\hline 3000 & 0.549 & 32.44614 & 0.58248 & 0.02735 & 0.001 & 0.04954 & 0.00092 & 45.15589 & 0.05018 & 0.00184 & 0.0224 & 0.00047 & 17.77885 & 0.51899 & 130.37 & 3.67 \\
\hline 3500 & 0.6371 & 25.87705 & 0.46904 & 0.02231 & 0.00097 & 0.03163 & 0.00055 & 36.1551 & 0.04093 & 0.00179 & 0.01979 & 0.00043 & 16.50248 & 0.411 & 121.32 & 2.92 \\
\hline 4000 & 0.8115 & 19.5607 & 0.26018 & 0.01729 & 0.00055 & 0.01616 & 0.00029 & 24.44639 & 0.03172 & 0.001 & 0.01448 & 0.00028 & 14.75656 & 0.21703 & 108.86 & 1.55 \\
\hline 5000 & 0.9605 & 17.68924 & 0.33206 & 0.01798 & 0.00052 & 0.00942 & 0.00027 & 15.74719 & 0.033 & 0.00095 & 0.01487 & 0.00034 & 14.87885 & 0.31476 & 109.73 & 2.25 \\
\hline 9000 & 1 & 18.20121 & 0.28389 & 0.03819 & 0.00155 & 0.01264 & 0.00085 & 20.53944 & 0.07008 & 0.00285 & 0.01512 & 0.00032 & 14.43957 & 0.34858 & 106.59 & 2.5 \\
\hline Integrated & & 58.95386 & 0.25342 & 0.2103 & 0.00187 & 0.14259 & 0.00093 & 71.47701 & 0.38593 & 0.00343 & 0.02192 & 0.00014 & 16.80943 & 0.26398 & 123.5 & 2.03 \\
\hline
\end{tabular}




\section{UM11-1682 BI\#L1}

Weighted average of $\mathrm{J}$ from standards $=4.215 \mathrm{e}-03+/-2.776 \mathrm{e}-05$

\begin{tabular}{|c|c|c|c|c|c|c|c|c|c|c|c|c|c|c|c|c|}
\hline $\begin{array}{l}\text { Laser Power } \\
(\mathrm{mW})\end{array}$ & $\begin{array}{l}\text { Cumulative } \\
\text { 39Ar }\end{array}$ & $\begin{array}{l}\text { 40Ar/39Ar } \\
\text { measured }\end{array}$ & $+/-$ & $\begin{array}{l}\text { 37Ar/39Ar } \\
\text { measured }\end{array}$ & $+/-$ & $\begin{array}{l}\text { 36Ar/39Ar } \\
\text { measured }\end{array}$ & $+/-$ & $\begin{array}{l}\% \text { Atmospheric } \\
40 \mathrm{Ar}\end{array}$ & $\mathrm{Ca} / \mathbf{K}$ & $+/-$ & $\mathrm{Cl} / \mathrm{K}$ & $+/-$ & $40^{* / 39 \mathrm{~K}}$ & $+/-$ & $\begin{array}{l}\text { Age } \\
\text { (Ma) }\end{array}$ & $\begin{array}{l}\text { +/- } \\
\text { (Ma) }\end{array}$ \\
\hline 300 & 0.0111 & 157.16371 & 2.67029 & 0.94185 & 0.02837 & 0.5096 & 0.00974 & 95.7843 & 1.72932 & 0.05212 & 0.02434 & 0.00157 & 6.62871 & 2.22412 & 49.71 & 16.45 \\
\hline 500 & 0.0325 & 70.2417 & 0.77737 & 14.98638 & 0.19473 & 0.21805 & 0.00569 & 90.01229 & 27.79208 & 0.36499 & 0.01944 & 0.00075 & 7.08761 & 1.71253 & 53.11 & 12.64 \\
\hline 700 & 0.0593 & 53.96235 & 0.64634 & 24.98179 & 0.3362 & 0.15719 & 0.00389 & 82.30848 & 46.66138 & 0.63923 & 0.02405 & 0.00077 & 9.71296 & 1.16385 & 72.39 & 8.5 \\
\hline 900 & 0.1129 & 42.0306 & 0.5149 & 16.28837 & 0.21373 & 0.10585 & 0.00261 & 71.27462 & 30.23471 & 0.40134 & 0.0295 & 0.0005 & 12.20542 & 0.81626 & 90.5 & 5.9 \\
\hline 1100 & 0.1786 & 32.81252 & 0.39948 & 19.1504 & 0.25109 & 0.07297 & 0.00151 & 60.95683 & 35.62009 & 0.47344 & 0.03205 & 0.00063 & 12.97509 & 0.50361 & 96.06 & 3.63 \\
\hline 1300 & 0.2496 & 26.2232 & 0.34589 & 10.56448 & 0.15208 & 0.0458 & 0.00129 & 48.34015 & 19.53008 & 0.28325 & 0.03202 & 0.00053 & 13.63335 & 0.42862 & 100.8 & 3.08 \\
\hline 1500 & 0.3268 & 24.78934 & 0.24833 & 7.62715 & 0.09379 & 0.03767 & 0.0009 & 42.42343 & 14.07057 & 0.17396 & 0.03223 & 0.00065 & 14.33305 & 0.32068 & 105.83 & 2.3 \\
\hline 1750 & 0.418 & 22.1039 & 0.2819 & 2.37238 & 0.03376 & 0.02921 & 0.00128 & 38.21787 & 4.36029 & 0.06216 & 0.03006 & 0.00039 & 13.66083 & 0.42904 & 101 & 3.08 \\
\hline 2000 & 0.4865 & 24.25814 & 0.25028 & 5.5103 & 0.05943 & 0.03672 & 0.00138 & 42.90935 & 10.15014 & 0.1099 & 0.03127 & 0.00055 & 13.88628 & 0.44473 & 102.62 & 3.19 \\
\hline 2250 & 0.5438 & 23.53837 & 0.21763 & 3.13055 & 0.03008 & 0.03161 & 0.00149 & 38.62948 & 5.75685 & 0.05543 & 0.03182 & 0.00047 & 14.4594 & 0.473 & 106.73 & 3.39 \\
\hline 2500 & 0.6001 & 21.38221 & 0.2175 & 4.89267 & 0.05525 & 0.02578 & 0.00141 & 33.78645 & 9.00851 & 0.10208 & 0.03227 & 0.00066 & 14.18734 & 0.45493 & 104.78 & 3.26 \\
\hline 3000 & 0.6741 & 20.76257 & 0.2484 & 5.87287 & 0.07519 & 0.02366 & 0.00093 & 31.38818 & 10.82079 & 0.13911 & 0.03299 & 0.00061 & 14.28453 & 0.33668 & 105.48 & 2.41 \\
\hline 3500 & 0.7408 & 19.14833 & 0.25879 & 4.67755 & 0.06373 & 0.01689 & 0.0018 & 24.08537 & 8.6111 & 0.11772 & 0.03207 & 0.00061 & 14.56202 & 0.57258 & 107.47 & 4.1 \\
\hline 4000 & 0.7981 & 18.58565 & 0.23526 & 1.84955 & 0.02247 & 0.01562 & 0.0014 & 24.05576 & 3.3981 & 0.04134 & 0.03059 & 0.0006 & 14.11063 & 0.4577 & 104.23 & 3.29 \\
\hline 5000 & 0.9521 & 17.59584 & 0.24563 & 2.52513 & 0.03539 & 0.01227 & 0.00054 & 19.4481 & 4.64153 & 0.06516 & 0.03078 & 0.00057 & 14.17518 & 0.26672 & 104.69 & 1.91 \\
\hline 9000 & 1 & 17.69575 & 0.20789 & 3.12299 & 0.03528 & 0.01434 & 0.00192 & 22.52909 & 5.74292 & 0.06502 & 0.02191 & 0.00045 & 13.71635 & 0.59814 & 101.4 & 4.3 \\
\hline Integrated & & 26.57037 & 0.09035 & 6.93609 & 0.02765 & 0.04606 & 0.00039 & 49.12937 & 12.78941 & 0.05123 & 0.03043 & 0.00016 & 13.56794 & 0.12856 & 100.33 & 1.13 \\
\hline
\end{tabular}




\section{UM11-1962 BI\#L1}

Weighted average of $\mathrm{J}$ from standards $=4.215 \mathrm{e}-03+/-2.776 \mathrm{e}-05$

\begin{tabular}{|c|c|c|c|c|c|c|c|c|c|c|c|c|c|c|c|c|}
\hline $\begin{array}{l}\text { Laser Power } \\
(\mathrm{mW})\end{array}$ & $\begin{array}{l}\text { Cumulative } \\
\text { 39Ar }\end{array}$ & $\begin{array}{l}40 \mathrm{Ar} / 39 \mathrm{Ar} \\
\text { measured }\end{array}$ & $+/-$ & $\begin{array}{l}\text { 37 Ar/39Ar } \\
\text { measured }\end{array}$ & $+/-$ & $\begin{array}{l}\text { 36Ar/39Ar } \\
\text { measured }\end{array}$ & $+/-$ & $\begin{array}{l}\text { \% Atmospheric } \\
40 \mathrm{Ar}\end{array}$ & $\mathrm{Ca} / \mathrm{K}$ & $+/-$ & $\mathrm{Cl} / \mathrm{K}$ & $+/-$ & $40^{* / 39 K}$ & $+/-$ & $\begin{array}{l}\text { Age } \\
\text { (Ma) }\end{array}$ & $\begin{array}{l}+/- \\
\text { (Ma) } \\
\end{array}$ \\
\hline 300 & 0.035 & 1056.44599 & 9.72531 & 0.05486 & 0.00278 & 3.4459 & 0.04579 & 96.38798 & 0.10067 & 0.00511 & 0.0267 & 0.00228 & 38.15949 & 11.6458 & 269.07 & 76.29 \\
\hline 500 & 0.0934 & 319.49377 & 3.62424 & 0.10556 & 0.00153 & 1.0203 & 0.0147 & 94.37407 & 0.1937 & 0.00281 & 0.02746 & 0.00102 & 17.97415 & 3.29192 & 131.75 & 23.27 \\
\hline 700 & 0.1773 & 156.63398 & 1.85312 & 0.47731 & 0.00688 & 0.46655 & 0.00665 & 88.00846 & 0.8761 & 0.01263 & 0.02781 & 0.00071 & 18.78559 & 1.43761 & 137.47 & 10.13 \\
\hline 900 & 0.2871 & 86.34363 & 1.11361 & 1.42142 & 0.02168 & 0.22878 & 0.00348 & 78.18979 & 2.61073 & 0.03986 & 0.02819 & 0.00049 & 18.84417 & 0.76026 & 137.89 & 5.36 \\
\hline 1100 & 0.4077 & 60.76327 & 0.74441 & 0.86832 & 0.01333 & 0.14332 & 0.00216 & 69.61398 & 1.59423 & 0.02449 & 0.028 & 0.00051 & 18.46585 & 0.53663 & 135.22 & 3.79 \\
\hline 1300 & 0.4967 & 49.3976 & 0.54387 & 0.03945 & 0.00088 & 0.10692 & 0.00148 & 63.9906 & 0.07239 & 0.00161 & 0.02747 & 0.00042 & 17.77758 & 0.41032 & 130.36 & 2.9 \\
\hline 1500 & 0.5749 & 44.30182 & 0.46271 & 0.04465 & 0.00132 & 0.08942 & 0.00123 & 59.67293 & 0.08192 & 0.00242 & 0.02734 & 0.00047 & 17.85422 & 0.36855 & 130.9 & 2.61 \\
\hline 1750 & 0.6646 & 38.04342 & 0.36993 & 0.02838 & 0.00074 & 0.06741 & 0.0008 & 52.39766 & 0.05207 & 0.00135 & 0.0274 & 0.00042 & 18.09578 & 0.27977 & 132.61 & 1.98 \\
\hline 2000 & 0.7416 & 33.79675 & 0.52468 & 0.02289 & 0.00097 & 0.05387 & 0.00069 & 47.1351 & 0.04199 & 0.00178 & 0.02743 & 0.00046 & 17.85121 & 0.43981 & 130.88 & 3.11 \\
\hline 2250 & 0.7881 & 31.98437 & 0.43649 & 0.03228 & 0.00146 & 0.04681 & 0.00083 & 43.27923 & 0.05923 & 0.00269 & 0.02771 & 0.00046 & 18.12535 & 0.42035 & 132.82 & 2.97 \\
\hline 2500 & 0.8225 & 31.20448 & 0.40579 & 0.04056 & 0.00154 & 0.04379 & 0.00105 & 41.50087 & 0.07442 & 0.00283 & 0.02811 & 0.00043 & 18.2375 & 0.42008 & 133.61 & 2.97 \\
\hline 3000 & 0.87 & 29.93584 & 0.45663 & 0.05395 & 0.00127 & 0.04085 & 0.00069 & 40.34452 & 0.099 & 0.00233 & 0.02867 & 0.00057 & 17.84133 & 0.38712 & 130.81 & 2.74 \\
\hline 3500 & 0.9048 & 30.53376 & 0.41896 & 0.05364 & 0.00183 & 0.04452 & 0.00117 & 43.11679 & 0.09843 & 0.00335 & 0.02798 & 0.00047 & 17.35234 & 0.46057 & 127.35 & 3.26 \\
\hline 4000 & 0.9292 & 28.34027 & 0.32551 & 0.06813 & 0.00295 & 0.03484 & 0.00113 & 36.34379 & 0.12502 & 0.00541 & 0.02987 & 0.00051 & 18.02231 & 0.41339 & 132.09 & 2.92 \\
\hline 5000 & 0.9629 & 25.64658 & 0.3257 & 0.13828 & 0.0023 & 0.02664 & 0.00078 & 30.68125 & 0.25375 & 0.00422 & 0.03088 & 0.00049 & 17.75904 & 0.35003 & 130.23 & 2.48 \\
\hline 9000 & 1 & 19.32533 & 0.26034 & 0.0614 & 0.00166 & 0.01371 & 0.00061 & 20.96963 & 0.11267 & 0.00304 & 0.02613 & 0.00056 & 15.25007 & 0.28863 & 112.39 & 2.06 \\
\hline Integrated & & 106.7573 & 0.37348 & 0.33637 & 0.0021 & 0.2978 & 0.00172 & 82.4279 & 0.61733 & 0.00385 & 0.02783 & 0.00017 & 18.75874 & 0.4839 & 137.29 & 3.52 \\
\hline
\end{tabular}




\section{UM11-2258 BI\#L1}

Weighted average of $\mathrm{J}$ from standards $=4.215 \mathrm{e}-03+/-2.776 \mathrm{e}-05$

\begin{tabular}{|c|c|c|c|c|c|c|c|c|c|c|c|c|c|c|c|c|}
\hline $\begin{array}{l}\text { Laser Power } \\
(\mathrm{mW})\end{array}$ & $\begin{array}{l}\text { Cumulative } \\
\text { 39Ar }\end{array}$ & $\begin{array}{l}\text { 40Ar/39Ar } \\
\text { measured }\end{array}$ & $+/-$ & $\begin{array}{l}\text { 37Ar/39Ar } \\
\text { measured }\end{array}$ & $+/-$ & $\begin{array}{l}\text { 36Ar/39Ar } \\
\text { measured }\end{array}$ & $+/-$ & $\begin{array}{l}\% \text { Atmospheric } \\
40 \mathrm{Ar}\end{array}$ & $\mathrm{Ca} / \mathrm{K}$ & $+/-$ & $\mathrm{Cl} / \mathrm{K}$ & $+/-$ & $40^{*} / 39 \mathrm{~K}$ & $+/-$ & $\begin{array}{l}\text { Age } \\
\text { (Ma) }\end{array}$ & $\begin{array}{l}\text { +/- } \\
\text { (Ma) }\end{array}$ \\
\hline 300 & 0.0228 & 87.11336 & 1.44508 & 0.08746 & 0.00341 & 0.27241 & 0.00474 & 92.42923 & 0.16048 & 0.00625 & 0.05644 & 0.00115 & 6.59331 & 1.46857 & 49.45 & 10.87 \\
\hline 500 & 0.0556 & 87.43382 & 1.63178 & 0.14319 & 0.00411 & 0.26995 & 0.00555 & 91.25123 & 0.26276 & 0.00754 & 0.04771 & 0.0011 & 7.64756 & 1.7071 & 57.24 & 12.58 \\
\hline 700 & 0.0894 & 43.06391 & 0.56261 & 0.0379 & 0.00318 & 0.10249 & 0.00145 & 70.36747 & 0.06955 & 0.00583 & 0.05069 & 0.00086 & 12.75246 & 0.50395 & 94.46 & 3.64 \\
\hline 900 & 0.1417 & 26.23573 & 0.3147 & 0.02386 & 0.00113 & 0.04008 & 0.00085 & 45.18414 & 0.04379 & 0.00207 & 0.05622 & 0.00085 & 14.3653 & 0.3323 & 106.06 & 2.38 \\
\hline 1100 & 0.2104 & 22.4945 & 0.2844 & 0.03894 & 0.00147 & 0.0252 & 0.00067 & 33.13765 & 0.07145 & 0.0027 & 0.05458 & 0.00068 & 15.0209 & 0.30414 & 110.75 & 2.18 \\
\hline 1300 & 0.2887 & 19.46476 & 0.23702 & 0.06 & 0.00141 & 0.01386 & 0.00053 & 21.05437 & 0.11009 & 0.00259 & 0.05476 & 0.0008 & 15.34378 & 0.26154 & 113.06 & 1.87 \\
\hline 1500 & 0.3533 & 18.78337 & 0.23321 & 0.12121 & 0.00324 & 0.01221 & 0.00066 & 19.18236 & 0.22242 & 0.00594 & 0.05411 & 0.00069 & 15.15758 & 0.28269 & 111.73 & 2.02 \\
\hline 1750 & 0.4233 & 18.94437 & 0.24906 & 0.40347 & 0.00477 & 0.0125 & 0.00043 & 19.34577 & 0.74051 & 0.00876 & 0.05299 & 0.00076 & 15.25984 & 0.25367 & 112.46 & 1.81 \\
\hline 2000 & 0.5035 & 19.05012 & 0.26347 & 1.58635 & 0.02306 & 0.01403 & 0.00056 & 21.10511 & 2.914 & 0.0424 & 0.05044 & 0.00082 & 15.023 & 0.27961 & 110.77 & 2 \\
\hline 2250 & 0.5685 & 17.56242 & 0.25266 & 2.11398 & 0.03128 & 0.00954 & 0.00064 & 15.08684 & 3.88466 & 0.05757 & 0.04938 & 0.00082 & 14.90988 & 0.29396 & 109.96 & 2.1 \\
\hline 2500 & 0.6267 & 17.13077 & 0.2136 & 0.42225 & 0.00548 & 0.00784 & 0.0005 & 13.3517 & 0.77501 & 0.01006 & 0.04826 & 0.00069 & 14.82221 & 0.24375 & 109.33 & 1.74 \\
\hline 3000 & 0.7208 & 16.76938 & 0.24317 & 0.03365 & 0.00079 & 0.00601 & 0.00038 & 10.58597 & 0.06175 & 0.00145 & 0.04825 & 0.00074 & 14.96798 & 0.25402 & 110.37 & 1.82 \\
\hline 3500 & 0.8005 & 16.73631 & 0.22819 & 0.03332 & 0.00169 & 0.00681 & 0.00048 & 12.02644 & 0.06113 & 0.00311 & 0.04635 & 0.00072 & 14.69774 & 0.25428 & 108.44 & 1.82 \\
\hline 4000 & 0.8689 & 16.60122 & 0.22925 & 0.05199 & 0.0011 & 0.00638 & 0.00056 & 11.35543 & 0.0954 & 0.00202 & 0.04838 & 0.00082 & 14.6903 & 0.27049 & 108.39 & 1.94 \\
\hline 5000 & 0.9729 & 16.95407 & 0.25861 & 0.06289 & 0.00156 & 0.00698 & 0.0004 & 12.16264 & 0.1154 & 0.00287 & 0.0439 & 0.00073 & 14.86658 & 0.26869 & 109.65 & 1.92 \\
\hline 9000 & 1 & 18.73992 & 0.16704 & 0.08754 & 0.00314 & 0.01313 & 0.00159 & 20.70235 & 0.16064 & 0.00576 & 0.04289 & 0.00071 & 14.83768 & 0.49369 & 109.44 & 3.53 \\
\hline Integrated & & 23.27621 & 0.09027 & 0.36053 & 0.00204 & 0.02994 & 0.00023 & 37.9263 & 0.66169 & 0.00374 & 0.05008 & 0.00021 & 14.43365 & 0.09825 & 106.55 & 0.98 \\
\hline
\end{tabular}




\section{SQ1-257 GL\#L1}

Weighted average of $\mathrm{J}$ from standards $=4.215 \mathrm{e}-03+/-2.776 \mathrm{e}-05$

\begin{tabular}{|c|c|c|c|c|c|c|c|c|c|c|c|c|c|c|c|c|}
\hline $\begin{array}{l}\text { Laser Power } \\
(\mathrm{mW})\end{array}$ & $\begin{array}{l}\text { Cumulative } \\
\text { 39Ar }\end{array}$ & $\begin{array}{l}\text { 40Ar/39Ar } \\
\text { measured }\end{array}$ & $+/-$ & $\begin{array}{l}\text { 37Ar/39Ar } \\
\text { measured }\end{array}$ & $+/-$ & $\begin{array}{l}\text { 36Ar/39Ar } \\
\text { measured }\end{array}$ & $+/-$ & $\begin{array}{l}\% \text { Atmospheric } \\
40 \mathrm{Ar}\end{array}$ & $\mathrm{Ca} / \mathrm{K}$ & $+/-$ & $\mathrm{Cl} / \mathrm{K}$ & $+/-$ & $40^{*} / 39 \mathrm{~K}$ & $+/-$ & $\begin{array}{l}\text { Age } \\
\text { (Ma) }\end{array}$ & $\begin{array}{l}+/- \\
\text { (Ma) }\end{array}$ \\
\hline 300 & 0.0016 & 64.89006 & 8.41683 & -0.39815 & 0.48919 & 0.13165 & 0.12412 & 60.03016 & -0.73035 & 0.89709 & -0.00896 & 0.01822 & 25.91729 & 37.04414 & 187.04 & 253.95 \\
\hline 500 & 0.0042 & 40.55807 & 5.12 & 0.19476 & 0.23463 & 0.1023 & 0.05919 & 74.54913 & 0.35741 & 0.43063 & 0.00145 & 0.00826 & 10.31624 & 17.88635 & 76.79 & 130.34 \\
\hline 750 & 0.0286 & 16.85776 & 0.37267 & 0.04836 & 0.03071 & 0.01156 & 0.00494 & 20.28195 & 0.08873 & 0.05635 & 0.00043 & 0.00094 & 13.41546 & 1.50166 & 99.23 & 10.81 \\
\hline 1000 & 0.0708 & 18.12552 & 0.25991 & 0.07493 & 0.01356 & 0.01746 & 0.00359 & 28.47485 & 0.1375 & 0.02489 & 0.00091 & 0.00061 & 12.94375 & 1.08034 & 95.84 & 7.79 \\
\hline 1500 & 0.1605 & 20.31068 & 0.40777 & 0.07814 & 0.00636 & 0.02385 & 0.00237 & 34.71474 & 0.14339 & 0.01168 & 0.00192 & 0.00029 & 13.24123 & 0.75458 & 97.98 & 5.43 \\
\hline 2000 & 0.2466 & 16.98027 & 0.27847 & 0.07583 & 0.00856 & 0.01183 & 0.00177 & 20.59009 & 0.13914 & 0.0157 & 0.0016 & 0.00036 & 13.46115 & 0.57327 & 99.56 & 4.13 \\
\hline 2500 & 0.3761 & 14.70354 & 0.22903 & 0.09611 & 0.00554 & 0.0066 & 0.00137 & 13.23937 & 0.17637 & 0.01016 & 0.0009 & 0.00017 & 12.73198 & 0.45532 & 94.31 & 3.29 \\
\hline 3000 & 0.521 & 15.24808 & 0.26248 & 0.11196 & 0.00447 & 0.00723 & 0.00116 & 13.97643 & 0.20545 & 0.0082 & 0.00115 & 0.00019 & 13.09243 & 0.41769 & 96.91 & 3.01 \\
\hline 4000 & 0.6864 & 14.79437 & 0.25456 & 0.20628 & 0.00595 & 0.00674 & 0.00098 & 13.36787 & 0.37856 & 0.01091 & 0.00071 & 0.00016 & 12.79281 & 0.37241 & 94.75 & 2.69 \\
\hline 5000 & 0.8467 & 14.82621 & 0.28577 & 0.21097 & 0.00671 & 0.00647 & 0.00094 & 12.79795 & 0.38716 & 0.01231 & 0.00083 & 0.00015 & 12.90479 & 0.38072 & 95.55 & 2.75 \\
\hline 6000 & 0.9515 & 14.18218 & 0.24863 & 0.15742 & 0.00699 & 0.0029 & 0.00175 & 5.97078 & 0.28888 & 0.01282 & 0.00145 & 0.00018 & 13.30895 & 0.56946 & 98.47 & 4.1 \\
\hline 9000 & 1 & 14.42617 & 0.24334 & 0.20413 & 0.01773 & 0.00316 & 0.00269 & 6.37109 & 0.3746 & 0.03254 & 0.00192 & 0.00043 & 13.4812 & 0.82953 & 99.71 & 5.97 \\
\hline Integrated & & 15.79202 & 0.09731 & 0.14075 & 0.00263 & 0.00916 & 0.00057 & 17.10177 & 0.25827 & 0.00482 & 0.00113 & 0.00009 & 13.06799 & 0.18966 & 96.73 & 1.5 \\
\hline
\end{tabular}




\section{GU1-736 PL\#L1}

Weighted average of $\mathrm{J}$ from standards $=4.215 \mathrm{e}-03+/-2.776 \mathrm{e}-05$

\begin{tabular}{|c|c|c|c|c|c|c|c|c|c|c|c|c|c|c|c|c|}
\hline $\begin{array}{l}\text { Laser Power } \\
\text { (mW) }\end{array}$ & $\begin{array}{l}\text { Cumulative } \\
\text { 39Ar }\end{array}$ & $\begin{array}{l}\text { 40Ar/39Ar } \\
\text { measured }\end{array}$ & $+/-$ & $\begin{array}{l}\begin{array}{l}37 \mathrm{Ar} / 39 \mathrm{Ar} \\
\text { measured }\end{array} \\
\end{array}$ & $+/-$ & $\begin{array}{l}\text { 36Ar/39Ar } \\
\text { measured }\end{array}$ & $+/-$ & $\begin{array}{l}\text { \% Atmospheric } \\
40 \mathrm{Ar}\end{array}$ & $\mathrm{Ca} / \mathrm{K}$ & $+/-$ & $\mathrm{Cl} / \mathrm{K}$ & $+/-$ & $40^{* / 39}$ & $+/-$ & $\begin{array}{l}\text { Age } \\
\text { (Ma) }\end{array}$ & $\begin{array}{l}+/- \\
\text { (Ma) }\end{array}$ \\
\hline 500 & 0.1174 & 413.69074 & 3.44286 & 211.68627 & 3.07223 & 1.40878 & 0.01988 & 96.41755 & 456.68821 & 7.793 & 0.01185 & 0.00145 & 17.42423 & 6.07748 & 127.86 & 43.05 \\
\hline 750 & 0.2881 & 111.74724 & 1.89714 & 198.06493 & 3.22608 & 0.39867 & 0.00622 & 90.83198 & 422.52284 & 8.00123 & 0.00871 & 0.00059 & 11.90837 & 2.34617 & 88.35 & 16.99 \\
\hline 1000 & 0.3861 & 80.45416 & 1.26491 & 235.80684 & 3.81515 & 0.3002 & 0.00471 & 86.1276 & 519.12273 & 10.07709 & 0.0108 & 0.00043 & 13.3868 & 1.58794 & 99.03 & 11.43 \\
\hline 1500 & 0.4556 & 80.58219 & 1.01094 & 267.39558 & 3.79063 & 0.30956 & 0.00516 & 86.1901 & 604.8539 & 10.57063 & 0.01199 & 0.00083 & 13.7149 & 1.89071 & 101.39 & 13.59 \\
\hline 2000 & 0.578 & 48.87929 & 0.70587 & 194.58829 & 3.00603 & 0.1902 & 0.00452 & 82.21144 & 413.92474 & 7.4131 & 0.01143 & 0.00057 & 10.07487 & 1.51248 & 75.03 & 11.03 \\
\hline 2500 & 0.6481 & 50.85938 & 0.65801 & 273.20022 & 3.99919 & 0.2146 & 0.0057 & 80.44365 & 621.12305 & 11.26578 & 0.01149 & 0.00065 & 12.3182 & 2.04064 & 91.32 & 14.75 \\
\hline 3000 & 0.7118 & 29.34262 & 0.41095 & 104.84209 & 1.38551 & 0.08926 & 0.00428 & 60.49489 & 207.75306 & 2.96503 & 0.00484 & 0.00069 & 12.507 & 1.37787 & 92.68 & 9.95 \\
\hline 5000 & 0.9031 & 21.29615 & 0.32055 & 35.90889 & 0.57699 & 0.04236 & 0.00158 & 44.93791 & 67.60222 & 1.1145 & 0.00168 & 0.00024 & 12.01485 & 0.50724 & 89.12 & 3.67 \\
\hline 9000 & 0.9935 & 18.94015 & 0.31173 & 26.28039 & 0.38269 & 0.03268 & 0.00302 & 39.61568 & 49.13279 & 0.72899 & 0.00218 & 0.00039 & 11.63522 & 0.94107 & 86.37 & 6.82 \\
\hline 9001 & 0.9945 & 273.18302 & 60.29012 & 96.55848 & 20.36786 & 1.13096 & 0.37202 & 119.43412 & 190.13717 & 43.04227 & 0.02608 & 0.03746 & -56.97019 & 93.6937 & -495.39 & 937.61 \\
\hline 9002 & 1 & 208.26904 & 9.96884 & 151.13867 & 7.18393 & 0.68814 & 0.06871 & 91.66604 & 310.45556 & 16.51986 & 0.01613 & 0.00804 & 19.42857 & 20.30461 & 142 & 142.71 \\
\hline Integrated & & 102.8521 & 0.52275 & 166.13989 & 0.98001 & 0.35658 & 0.00278 & 89.15385 & 345.36576 & 2.30802 & 0.00792 & 0.00024 & 12.63513 & 0.90091 & 93.61 & 6.53 \\
\hline
\end{tabular}




\section{GU1-1165 BI\#L1}

Weighted average of $\mathrm{J}$ from standards $=4.215 \mathrm{e}-03+/-2.776 \mathrm{e}-05$

\begin{tabular}{|c|c|c|c|c|c|c|c|c|c|c|c|c|c|c|c|c|}
\hline $\begin{array}{l}\text { Laser Power } \\
(\mathrm{mW})\end{array}$ & $\begin{array}{l}\text { Cumulative } \\
\text { 39Ar }\end{array}$ & $\begin{array}{l}\text { 40Ar/39Ar } \\
\text { measured }\end{array}$ & $+/-$ & $\begin{array}{l}\text { 37Ar/39Ar } \\
\text { measured }\end{array}$ & $+/-$ & $\begin{array}{l}\text { 36Ar/39Ar } \\
\text { measured }\end{array}$ & $+/-$ & $\begin{array}{l}\text { \% Atmospheric } \\
40 \mathrm{Ar}\end{array}$ & $\mathrm{Ca} / \mathrm{K}$ & $+/-$ & $\mathrm{Cl} / \mathrm{K}$ & $+/-$ & $40^{* / 39 \mathrm{~K}}$ & $+/-$ & $\begin{array}{l}\text { Age } \\
\text { (Ma) }\end{array}$ & $\begin{array}{l}+/- \\
\text { (Мa) }\end{array}$ \\
\hline 300 & 0.0118 & 5230.2803 & 76.58857 & 0.06655 & 0.00468 & 16.91429 & 0.22767 & 95.56265 & 0.12211 & 0.00858 & 0.07498 & 0.00835 & 232.09568 & 59.33086 & 1230.79 & 228.06 \\
\hline 500 & 0.0455 & 677.18997 & 7.75008 & 0.08265 & 0.00116 & 2.14498 & 0.02036 & 93.6021 & 0.15166 & 0.00213 & 0.06559 & 0.00153 & 43.32656 & 7.42312 & 302.6 & 47.73 \\
\hline 700 & 0.1094 & 246.34788 & 3.29156 & 0.12717 & 0.00156 & 0.74467 & 0.01004 & 89.33176 & 0.23336 & 0.00286 & 0.06065 & 0.00121 & 26.28017 & 3.0489 & 189.53 & 20.87 \\
\hline 900 & 0.1872 & 105.87246 & 1.60217 & 0.04823 & 0.00107 & 0.28898 & 0.00473 & 80.67553 & 0.08849 & 0.00196 & 0.05793 & 0.0012 & 20.45425 & 1.37085 & 149.19 & 9.6 \\
\hline 1100 & 0.272 & 66.67316 & 1.01185 & 0.01885 & 0.0005 & 0.16075 & 0.00246 & 71.27703 & 0.03458 & 0.00092 & 0.05641 & 0.00111 & 19.14224 & 0.78865 & 139.99 & 5.55 \\
\hline 1300 & 0.3633 & 48.05003 & 0.77019 & 0.01918 & 0.00055 & 0.09978 & 0.00167 & 61.39789 & 0.03519 & 0.00102 & 0.05616 & 0.00107 & 18.53711 & 0.58657 & 135.72 & 4.14 \\
\hline 1500 & 0.4391 & 40.1363 & 0.63747 & 0.01985 & 0.00044 & 0.07415 & 0.00126 & 54.62981 & 0.03643 & 0.00081 & 0.05521 & 0.00105 & 18.1967 & 0.49741 & 133.32 & 3.51 \\
\hline 1750 & 0.529 & 35.90989 & 0.57729 & 0.02286 & 0.00049 & 0.05938 & 0.00106 & 48.89985 & 0.04195 & 0.0009 & 0.05483 & 0.00108 & 18.33513 & 0.45834 & 134.3 & 3.24 \\
\hline 2000 & 0.612 & 32.32453 & 0.51601 & 0.03192 & 0.00089 & 0.04945 & 0.00081 & 45.24152 & 0.05856 & 0.00164 & 0.05406 & 0.001 & 17.68456 & 0.40291 & 129.7 & 2.85 \\
\hline 2250 & 0.688 & 30.02495 & 0.48072 & 0.04371 & 0.00074 & 0.04169 & 0.00074 & 41.05488 & 0.0802 & 0.00135 & 0.05391 & 0.00104 & 17.68128 & 0.38826 & 129.68 & 2.75 \\
\hline 2500 & 0.7612 & 25.64589 & 0.38401 & 0.04483 & 0.00069 & 0.02781 & 0.00039 & 32.07119 & 0.08226 & 0.00126 & 0.05352 & 0.00097 & 17.40133 & 0.31924 & 127.69 & 2.26 \\
\hline 3000 & 0.8512 & 22.66105 & 0.35912 & 0.06594 & 0.00111 & 0.02097 & 0.00034 & 27.35705 & 0.121 & 0.00204 & 0.05226 & 0.00099 & 16.44085 & 0.30317 & 120.88 & 2.16 \\
\hline 3500 & 0.9205 & 21.26953 & 0.32455 & 0.081 & 0.00136 & 0.01682 & 0.00034 & 23.37362 & 0.14863 & 0.00249 & 0.05234 & 0.00096 & 16.27625 & 0.29002 & 119.71 & 2.06 \\
\hline 4000 & 0.9498 & 19.56367 & 0.35146 & 0.04415 & 0.00119 & 0.0134 & 0.00043 & 20.25849 & 0.08101 & 0.00219 & 0.04899 & 0.00099 & 15.57717 & 0.33078 & 114.72 & 2.36 \\
\hline 5000 & 0.9771 & 18.33706 & 0.31793 & 0.06535 & 0.00135 & 0.0103 & 0.00047 & 16.60123 & 0.11992 & 0.00247 & 0.04889 & 0.00098 & 15.26882 & 0.31863 & 112.52 & 2.28 \\
\hline 9000 & 1 & 18.03563 & 0.32053 & 0.15225 & 0.00324 & 0.01045 & 0.00048 & 17.07875 & 0.27939 & 0.00594 & 0.04895 & 0.00094 & 14.93235 & 0.3198 & 110.12 & 2.29 \\
\hline Integrated & & 136.77578 & 0.78238 & 0.04973 & 0.00026 & 0.38926 & 0.00207 & 84.11332 & 0.09125 & 0.00047 & 0.05523 & 0.00031 & 21.72518 & 0.78664 & 158.07 & 5.57 \\
\hline
\end{tabular}




\section{GU1-2840 BI\#L1}

Weighted average of $\mathrm{J}$ from standards $=4.215 \mathrm{e}-03+/-2.776 \mathrm{e}-05$

\begin{tabular}{|c|c|c|c|c|c|c|c|c|c|c|c|c|c|c|c|c|}
\hline $\begin{array}{l}\text { Laser Power } \\
(\mathrm{mW})\end{array}$ & $\begin{array}{l}\text { Cumulative } \\
\text { 39Ar }\end{array}$ & $\begin{array}{l}\text { 40Ar/39Ar } \\
\text { measured }\end{array}$ & $+/-$ & $\begin{array}{l}\text { 37Ar/39Ar } \\
\text { measured }\end{array}$ & $+/-$ & $\begin{array}{l}\text { 36Ar/39Ar } \\
\text { measured }\end{array}$ & $+/-$ & $\begin{array}{l}\% \text { Atmospheric } \\
40 \mathrm{Ar}\end{array}$ & $\mathrm{Ca} / \mathrm{K}$ & $+/-$ & $\mathrm{Cl} / \mathrm{K}$ & $+/-$ & $40^{*} / 39 \mathrm{~K}$ & $+/-$ & $\begin{array}{l}\text { Age } \\
\text { (Ma) }\end{array}$ & $\begin{array}{l}+/- \\
\text { (Ma) }\end{array}$ \\
\hline 300 & 0.0125 & 109.13816 & 2.32525 & 0.08515 & 0.00343 & 0.3438 & 0.00669 & 93.10483 & 0.15625 & 0.00629 & 0.01906 & 0.00075 & 7.52367 & 2.09476 & 56.32 & 15.44 \\
\hline 500 & 0.0311 & 75.54401 & 1.40082 & 0.11009 & 0.00272 & 0.21556 & 0.00384 & 84.33957 & 0.20201 & 0.005 & 0.02788 & 0.00068 & 11.82679 & 1.33543 & 87.76 & 9.67 \\
\hline 700 & 0.0603 & 36.99806 & 0.63282 & 0.41213 & 0.00705 & 0.0795 & 0.00124 & 63.45647 & 0.75643 & 0.01294 & 0.03099 & 0.00066 & 13.51348 & 0.55611 & 99.94 & 4 \\
\hline 900 & 0.0973 & 25.88192 & 0.4498 & 0.11936 & 0.00189 & 0.03886 & 0.00069 & 44.37977 & 0.21903 & 0.00347 & 0.03331 & 0.00075 & 14.38028 & 0.38528 & 106.16 & 2.76 \\
\hline 1100 & 0.1478 & 22.05782 & 0.39465 & 0.1333 & 0.00254 & 0.02561 & 0.00051 & 34.30314 & 0.2446 & 0.00466 & 0.03348 & 0.00061 & 14.47315 & 0.34342 & 106.83 & 2.46 \\
\hline 1300 & 0.2037 & 19.5923 & 0.3303 & 0.1203 & 0.00226 & 0.0167 & 0.0004 & 25.1755 & 0.22075 & 0.00416 & 0.03436 & 0.00058 & 14.63886 & 0.3092 & 108.02 & 2.21 \\
\hline 1500 & 0.2687 & 18.61664 & 0.32954 & 0.09621 & 0.00187 & 0.01333 & 0.00036 & 21.14716 & 0.17654 & 0.00343 & 0.03465 & 0.00069 & 14.65732 & 0.30933 & 108.15 & 2.22 \\
\hline 1750 & 0.3577 & 17.73877 & 0.32262 & 0.08922 & 0.00207 & 0.01114 & 0.00023 & 18.54855 & 0.16372 & 0.0038 & 0.03451 & 0.00065 & 14.4252 & 0.29929 & 106.49 & 2.15 \\
\hline 2000 & 0.4523 & 17.22047 & 0.30365 & 0.09322 & 0.0016 & 0.00924 & 0.00021 & 15.84606 & 0.17106 & 0.00293 & 0.03468 & 0.00069 & 14.46766 & 0.28671 & 106.79 & 2.05 \\
\hline 2250 & 0.5517 & 16.85825 & 0.32726 & 0.09768 & 0.00207 & 0.00845 & 0.0002 & 14.78682 & 0.17923 & 0.0038 & 0.03459 & 0.00074 & 14.34114 & 0.30609 & 105.88 & 2.19 \\
\hline 2500 & 0.6374 & 16.83192 & 0.30509 & 0.11775 & 0.00206 & 0.00786 & 0.0002 & 13.77305 & 0.21607 & 0.00378 & 0.03502 & 0.00071 & 14.48924 & 0.28941 & 106.94 & 2.07 \\
\hline 3000 & 0.7555 & 16.4006 & 0.21504 & 0.10526 & 0.00181 & 0.00719 & 0.00015 & 12.91708 & 0.19316 & 0.00333 & 0.03505 & 0.00065 & 14.25732 & 0.19441 & 105.28 & 1.39 \\
\hline 3500 & 0.845 & 16.20337 & 0.28892 & 0.11099 & 0.00193 & 0.00651 & 0.00018 & 11.84657 & 0.20366 & 0.00354 & 0.03501 & 0.00071 & 14.25877 & 0.27662 & 105.29 & 1.98 \\
\hline 4000 & 0.8926 & 16.10583 & 0.26652 & 0.15347 & 0.00251 & 0.00655 & 0.00032 & 11.95752 & 0.28164 & 0.00461 & 0.03425 & 0.0006 & 14.15536 & 0.26749 & 104.55 & 1.92 \\
\hline 5000 & 0.9669 & 15.51052 & 0.28734 & 0.14502 & 0.00307 & 0.00537 & 0.0002 & 10.16676 & 0.26612 & 0.00564 & 0.0341 & 0.00074 & 13.90835 & 0.27741 & 102.78 & 1.99 \\
\hline 9000 & 1 & 18.28359 & 0.26884 & 0.61712 & 0.01018 & 0.01436 & 0.00051 & 22.96702 & 1.13282 & 0.0187 & 0.03207 & 0.0006 & 14.06765 & 0.27975 & 103.92 & 2.01 \\
\hline Integrated & & 20.46422 & 0.09705 & 0.13672 & 0.00071 & 0.02116 & 0.00012 & 30.54076 & 0.25089 & 0.00131 & 0.03405 & 0.00019 & 14.19503 & 0.08677 & 104.84 & 0.92 \\
\hline
\end{tabular}




\section{GU1-2845 PL\#L1}

Weighted average of $\mathrm{J}$ from standards $=4.215 \mathrm{e}-03+/-2.776 \mathrm{e}-05$

\begin{tabular}{|c|c|c|c|c|c|c|c|c|c|c|c|c|c|c|c|c|}
\hline $\begin{array}{l}\text { Laser Power } \\
(\mathrm{mW})\end{array}$ & $\begin{array}{l}\text { Cumulative } \\
\text { 39Ar }\end{array}$ & $\begin{array}{l}\text { 40Ar/39Ar } \\
\text { measured }\end{array}$ & $+/-$ & $\begin{array}{l}\text { 37Ar/39Ar } \\
\text { measured }\end{array}$ & $+/-$ & $\begin{array}{l}\text { 36Ar/39Ar } \\
\text { measured }\end{array}$ & $+/-$ & $\begin{array}{l}\% \text { Atmospheric } \\
40 \mathrm{Ar}\end{array}$ & $\mathrm{Ca} / \mathrm{K}$ & $+/-$ & $\mathrm{Cl} / \mathrm{K}$ & $+/-$ & $40^{*} / 39 \mathrm{~K}$ & $+/-$ & $\begin{array}{l}\text { Age } \\
\text { (Ma) }\end{array}$ & $\begin{array}{l}+/- \\
\text { (Ma) }\end{array}$ \\
\hline 500 & 0.2284 & 1506.8979 & 8.36052 & 290.77097 & 3.77925 & 5.00892 & 0.06512 & 96.63493 & 671.39309 & 10.98129 & 0.06475 & 0.00487 & 63.81067 & 24.48702 & 429.73 & 146.74 \\
\hline 750 & 0.3286 & 223.70167 & 3.35421 & 1053.95082 & 8.45509 & 1.00577 & 0.01582 & 94.02377 & 7563.44281 & 237.30827 & 0.15238 & 0.00348 & 52.28507 & 20.29442 & 359.3 & 126.45 \\
\hline 1000 & 0.4564 & 262.40492 & 2.25009 & 1103.90566 & 9.44215 & 1.14039 & 0.01554 & 93.74601 & 9189.94303 & 356.63917 & 0.22419 & 0.00602 & 74.4553 & 18.45976 & 492.41 & 106.84 \\
\hline 1500 & 0.6607 & 285.63835 & 2.85449 & 981.31281 & 9.79344 & 1.17347 & 0.01636 & 93.08246 & 5865.39643 & 190.6828 & 0.14261 & 0.0041 & 64.36362 & 12.78702 & 433.04 & 76.48 \\
\hline 2000 & 0.7328 & 316.1236 & 2.96601 & 1179.84763 & 11.04266 & 1.32485 & 0.01651 & 93.07821 & 12980.76715 & 728.48216 & 0.37458 & 0.00725 & 131.20165 & 24.45068 & 794.15 & 119.72 \\
\hline 2500 & 0.8044 & 119.67323 & 1.89617 & 1033.99954 & 18.11864 & 0.66901 & 0.01014 & 93.97707 & 7032.71804 & 456.80172 & 0.16749 & 0.00407 & 26.71623 & 9.16468 & 192.51 & 62.64 \\
\hline 3000 & 0.8849 & 71.43579 & 0.99242 & 525.73495 & 8.10141 & 0.34107 & 0.00939 & 80.44067 & 1534.3141 & 37.60557 & 0.05802 & 0.00244 & 22.21776 & 4.14214 & 161.5 & 28.8 \\
\hline 5000 & 0.973 & 73.13827 & 1.01363 & 546.73503 & 8.80818 & 0.34898 & 0.00775 & 79.39878 & 1634.14832 & 42.88563 & 0.09783 & 0.00255 & 24.53808 & 3.36345 & 177.56 & 23.18 \\
\hline 9000 & 0.9995 & 59.99065 & 4.30719 & 12.68789 & 0.72887 & 0.11975 & 0.0311 & 57.26845 & 23.49102 & 1.36166 & 0.02301 & 0.00647 & 25.85402 & 9.88914 & 186.61 & 67.81 \\
\hline 9001 & 1 & 800.98446 & 1744.89473 & 46.44072 & 101.41057 & 1.50259 & 3.69991 & 54.95788 & 88.10181 & 198.90768 & -0.16087 & 0.41812 & 373.00078 & 1004.99428 & 1704.42 & 2971.06 \\
\hline Integrated & & 360.49525 & 1.47194 & 931.27419 & 3.72418 & 1.41173 & 0.00907 & 94.4284 & 4991.69647 & 58.31342 & 0.14066 & 0.00174 & 58.67241 & 7.17414 & 398.67 & 43.8 \\
\hline
\end{tabular}




\section{GU2-1397 BI\#L1}

Weighted average of $\mathrm{J}$ from standards $=4.215 \mathrm{e}-03+/-2.776 \mathrm{e}-05$

\begin{tabular}{|c|c|c|c|c|c|c|c|c|c|c|c|c|c|c|c|c|}
\hline $\begin{array}{l}\text { Laser Power } \\
(\mathrm{mW})\end{array}$ & $\begin{array}{l}\text { Cumulative } \\
\text { 39Ar }\end{array}$ & $\begin{array}{l}\text { 40Ar/39Ar } \\
\text { measured }\end{array}$ & $+/-$ & $\begin{array}{l}\text { 37Ar/39Ar } \\
\text { measured }\end{array}$ & $+/-$ & $\begin{array}{l}\text { 36Ar/39Ar } \\
\text { measured }\end{array}$ & $+/-$ & $\begin{array}{l}\% \text { Atmospheric } \\
40 \mathrm{Ar}\end{array}$ & $\mathrm{Ca} / \mathrm{K}$ & $+/-$ & $\mathrm{Cl} / \mathrm{K}$ & $+/-$ & $40^{*} / 39 \mathrm{~K}$ & $+/-$ & $\begin{array}{l}\text { Age } \\
\text { (Ma) }\end{array}$ & $\begin{array}{l}+/- \\
\text { (Ma) }\end{array}$ \\
\hline 300 & 0.0183 & 73.2399 & 2.1046 & 0.04273 & 0.06314 & 0.19969 & 0.01451 & 80.59788 & 0.0784 & 0.11585 & 0.01233 & 0.00212 & 14.20476 & 4.18552 & 104.91 & 30.03 \\
\hline 500 & 0.0233 & 102.88813 & 9.89131 & -0.11618 & 0.22831 & 0.27419 & 0.06726 & 78.78188 & -0.21316 & 0.41884 & 0.00956 & 0.0102 & 21.82284 & 19.36497 & 158.75 & 134.85 \\
\hline 750 & 0.0359 & 83.61474 & 3.60648 & 0.77379 & 0.09219 & 0.25806 & 0.02324 & 91.15774 & 1.42057 & 0.16934 & 0.03055 & 0.00295 & 7.39485 & 6.4249 & 55.37 & 47.38 \\
\hline 1000 & 0.0608 & 64.62586 & 1.67622 & 0.12103 & 0.03687 & 0.19157 & 0.01175 & 87.61873 & 0.22208 & 0.06767 & 0.02736 & 0.00276 & 7.99851 & 3.31902 & 59.82 & 24.42 \\
\hline 1250 & 0.0732 & 30.434 & 2.37046 & 0.34111 & 0.09146 & 0.10369 & 0.02615 & 100.68872 & 0.62603 & 0.1679 & 0.0069 & 0.00366 & -0.20945 & 7.85388 & -1.59 & 59.77 \\
\hline 1500 & 0.0945 & 27.45438 & 1.16619 & 0.21192 & 0.04261 & 0.04992 & 0.01209 & 53.72857 & 0.3889 & 0.0782 & 0.01054 & 0.00214 & 12.69169 & 3.71123 & 94.02 & 26.79 \\
\hline 1750 & 0.113 & 31.61663 & 1.64506 & 0.13848 & 0.04842 & 0.06341 & 0.01338 & 59.28913 & 0.25412 & 0.08887 & 0.01332 & 0.0022 & 12.86057 & 4.10149 & 95.24 & 29.58 \\
\hline 2000 & 0.1384 & 34.9334 & 1.16342 & 0.01627 & 0.00645 & 0.05606 & 0.00862 & 47.45384 & 0.02985 & 0.01183 & 0.01605 & 0.00235 & 18.34077 & 2.67385 & 134.34 & 18.87 \\
\hline 2500 & 0.1763 & 30.34656 & 0.71655 & 0.01431 & 0.0046 & 0.04724 & 0.0067 & 46.04356 & 0.02626 & 0.00843 & 0.01385 & 0.00078 & 16.35806 & 2.05136 & 120.29 & 14.59 \\
\hline 3000 & 0.2409 & 26.10801 & 0.47985 & 0.11119 & 0.02041 & 0.04626 & 0.00415 & 52.38825 & 0.20403 & 0.03746 & 0.01787 & 0.00104 & 12.41732 & 1.26045 & 92.04 & 9.11 \\
\hline 4000 & 0.5685 & 25.41297 & 0.50414 & 0.05714 & 0.00367 & 0.04295 & 0.00108 & 49.98312 & 0.10485 & 0.00673 & 0.01416 & 0.00042 & 12.69643 & 0.45886 & 94.05 & 3.31 \\
\hline 5000 & 0.8003 & 21.22698 & 0.38063 & 0.07252 & 0.00616 & 0.02633 & 0.00115 & 36.67034 & 0.13307 & 0.01131 & 0.02379 & 0.00065 & 13.42485 & 0.43388 & 99.3 & 3.12 \\
\hline 9000 & 1 & 18.40759 & 0.29455 & 0.03301 & 0.00603 & 0.01696 & 0.00144 & 27.25483 & 0.06057 & 0.01107 & 0.03133 & 0.00062 & 13.36934 & 0.48988 & 98.9 & 3.53 \\
\hline Integrated & & 26.70789 & 0.22909 & 0.07451 & 0.00379 & 0.04632 & 0.00102 & 51.28865 & 0.13673 & 0.00695 & 0.02039 & 0.00029 & 12.99599 & 0.33106 & 96.21 & 2.47 \\
\hline
\end{tabular}




\section{UM2-C2-311 BI\#1}

Weighted average of $\mathrm{J}$ from standards $=3.730 \mathrm{e}-03+/-1.543$

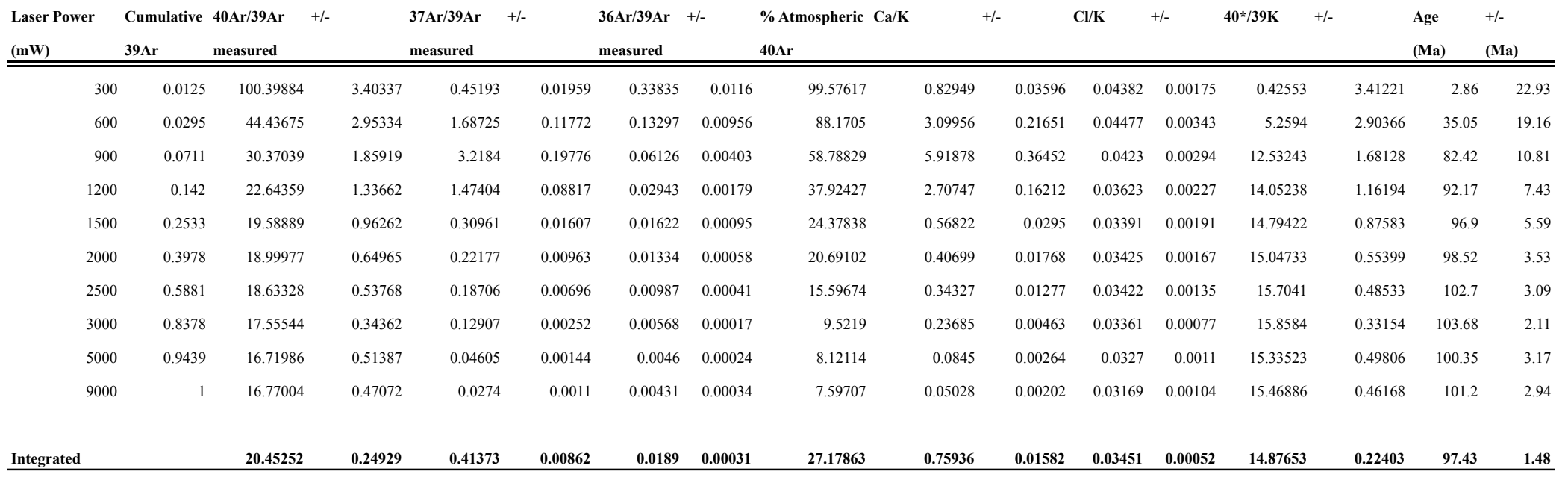




\section{UM2-C2-311 BI\#2}

Weighted average of $\mathrm{J}$ from standards $=3.730 \mathrm{e}-03+/-1.543$

\begin{tabular}{|c|c|c|c|c|c|c|c|c|c|c|c|c|c|c|c|c|}
\hline $\begin{array}{l}\text { Laser Power } \\
(\mathrm{mW})\end{array}$ & $\begin{array}{l}\text { Cumulative } \\
\text { 39Ar }\end{array}$ & $\begin{array}{l}40 \mathrm{Ar} / 39 \mathrm{Ar} \\
\text { measured }\end{array}$ & $+/-$ & $\begin{array}{l}\begin{array}{l}37 \mathrm{Ar} / 39 \mathrm{Ar} \\
\text { measured }\end{array} \\
\end{array}$ & & $\begin{array}{l}\text { 36Ar/39Ar } \\
\text { measured }\end{array}$ & $+/-$ & $\begin{array}{l}\% \text { Atmospheric } \mathrm{Ca} / \mathrm{K} \\
40 \mathrm{Ar}\end{array}$ & & & $\mathrm{Cl} / \mathbf{K}$ & $+/-$ & $40^{* / 39 \mathrm{~K}}$ & & $\begin{array}{l}\text { Age } \\
\text { (Ma) }\end{array}$ & $\begin{array}{l}+/- \\
\text { (Ma) }\end{array}$ \\
\hline 300 & 0.0245 & 53.14155 & 3.35258 & 2.28169 & 0.13508 & 0.17192 & 0.0105 & 95.29582 & 4.19334 & 0.24866 & 0.03193 & 0.00243 & 2.50251 & 3.28797 & 16.76 & 21.92 \\
\hline 600 & 0.067 & 24.83539 & 1.65455 & 2.90005 & 0.172 & 0.04301 & 0.00282 & 50.27155 & 5.33211 & 0.31689 & 0.03528 & 0.00229 & 12.36083 & 1.50045 & 81.32 & 9.65 \\
\hline 900 & 0.1411 & 20.43266 & 1.25622 & 1.9435 & 0.10886 & 0.02025 & 0.00123 & 28.54749 & 3.57095 & 0.20028 & 0.03373 & 0.00207 & 14.59849 & 1.14506 & 95.66 & 7.31 \\
\hline 1200 & 0.2738 & 18.65038 & 0.96392 & 0.69912 & 0.03394 & 0.01219 & 0.00071 & 19.03801 & 1.28342 & 0.06235 & 0.03293 & 0.00183 & 15.08313 & 0.89576 & 98.75 & 5.71 \\
\hline 1500 & 0.392 & 18.11299 & 0.72286 & 0.42408 & 0.01846 & 0.00955 & 0.0005 & 15.41019 & 0.77835 & 0.0339 & 0.03285 & 0.00147 & 15.30121 & 0.67606 & 100.13 & 4.3 \\
\hline 2000 & 0.5861 & 17.49564 & 0.44842 & 0.07072 & 0.00251 & 0.0064 & 0.00027 & 10.80191 & 0.12977 & 0.00461 & 0.03333 & 0.00115 & 15.58006 & 0.41841 & 101.91 & 2.66 \\
\hline 2500 & 0.826 & 17.31553 & 0.34898 & 0.05897 & 0.00133 & 0.00472 & 0.00016 & 8.04809 & 0.1082 & 0.00244 & 0.03409 & 0.00089 & 15.89532 & 0.33544 & 103.91 & 2.13 \\
\hline 3000 & 0.9348 & 16.90369 & 0.53321 & 0.04631 & 0.00135 & 0.00453 & 0.00036 & 7.91431 & 0.08498 & 0.00248 & 0.03389 & 0.00117 & 15.53904 & 0.52342 & 101.65 & 3.33 \\
\hline 5000 & 0.9912 & 16.76691 & 0.61235 & 0.06718 & 0.00291 & 0.00524 & 0.00043 & 9.21823 & 0.12327 & 0.00534 & 0.03248 & 0.00123 & 15.19506 & 0.59671 & 99.46 & 3.8 \\
\hline 9000 & 1 & 22.83849 & 0.74537 & 0.04112 & 0.00688 & 0.01987 & 0.00255 & 25.73059 & 0.07545 & 0.01263 & 0.03996 & 0.00177 & 16.94045 & 0.99461 & 110.54 & 6.3 \\
\hline Integrated & & 19.02537 & 0.24962 & 0.50353 & 0.00971 & 0.01364 & 0.00028 & 20.99682 & 0.92423 & 0.01783 & 0.03355 & 0.00049 & 15.01253 & 0.23547 & 98.3 & 1.55 \\
\hline
\end{tabular}

